\title{
FERRAMENTA WEB PARA CRIAÇÃO, DISPONIBILIZAÇÃO, DESCRIÇÃO DE METADADOS OBAA E EMPACOTAMENTO SCORM DE OBJETOS DE APRENDIZAGEM
}

\author{
Lucas Ferrari da Costa, IFRS Bento Gonçalves, \\ lukas.ferrari95@gmail.com \\ Maximiliano Reidel, IFRS Bento Gonçalves, \\ maximiliano.reidel@bento.ifrs.edu.br \\ Vinícius de Carli, IFRS Bento Gonçalves, \\ vinidcali@gmail.com
}

Júlia Marques Carvalho da Silva, IFRS Bento Gonçalves, julia.silva@bento.ifrs.edu.br

\begin{abstract}
Resumo. Este artigo apresenta o desenvolvimento de uma ferramenta de autoria para objetos de aprendizagem (OAs) com preenchimento de metadados no padrão OBAA e empacotamento SCORM. A escolha das especificações dá-se pela inexistência de ferramentas que combinem ambas tecnologias. Espera-se, com a utilização da aplicação, facilitar, para o professor, a criação de conteúdos educacionais e incentivar os alunos a criarem seus próprios materiais, bem como disseminar o modelo OBAA. Em fase de desenvolvimento inicial, não é possível, ainda, validar o sistema. Entretanto, a partir de OAs-teste é plausível destacar que, até então, a ferramenta apresenta-se fiel aos objetivos iniciais, funcionando conforme planejado.
\end{abstract}

Palavras-chave. ferramenta de autoria, repositório educacional, informática na educação, padrões.

\section{WEB TOOL FOR CREATING, SUPPLYING, OBAA METADATA DESCRIBING AND SCORM PACKING LEARNING OBJECTS}

\begin{abstract}
This paper presents a learning objects integrated tool development. It allows filling metadata in the OBAA standard and packaging using SCORM. Those specifications where chosen because there are no tools which combine both technologies. We hope, by using the application, to facilitate, to the teacher, the creation of educational content and to encourage students to create their own materials, as well as to disseminate the OBAA model. In
\end{abstract}


early development stage, it is not possible, yet, to validate the system. However, from test LOs it is plausible to note that, so far, the tool has been true to the original goals, working as planned.

Keywords. authoring tool, educational repository, education informatics, standards.

\section{Introdução}

Objetos de Aprendizagem (OAs) são conjuntos de conteúdos educacionais com um arquivo de metadados (Wiley, 2000). Os metadados ajudam a descrever o OA: como ele pode ser aplicado ou o que é necessário para sua execução (Silva, 2011). Eles também permitem que o OA seja compartilhado em repositórios educacionais. Entretanto, ambos repositório e metadados precisam seguir o mesmo padrão, como o IEEE LOM, o CanCore ou o OBAA. O OBAA é o modelo brasileiro que estende o LOM, adicionando novas categorias como interoperabilidade e acessibilidade.

A fim de simplificar a geração de OAs e de seus metadados, foram criadas ferramentas de autoria. Elas são "recursos amigáveis para leigos ou nãoprogramadores" capazes de prover a estrutura essencial para organizar e editar elementos em um software multimídia (Nienow e Bez, 2009). Entretanto, a maioria delas é complexa por necessitar conhecimentos de Informática ou instalações adicionais para serem usadas, ou requerem pagamento. Ainda, são poucas que permitem a criação de objetos no padrão OBAA ou fazem uso do SCORM quanto ao empacotamento de arquivos.

A presente pesquisa, conduzida com foco nos conceitos básicos relevantes e na situação atual do tema, bem como nas tecnologias necessárias para a criação do software, caracteriza-se como preparação para a fase de construção, a qual gerou uma ferramenta - no momento em estágio inicial de desenvolvimento - de simples e fácil procedimento, que, além de padronizar a descrição de metadados e o empacotamento do $\mathrm{OA}$, exige apenas um navegador com acesso à Internet para funcionar. Também buscou-se adaptar a ferramenta para diferentes dispositivos e internacionalizá-la. Espera-se com seu uso facilitar a tarefa do professor de criar conteúdos educacionais, encorajar estudantes a inventar seus próprios materiais e disseminar o modelo OBAA.

Este documento está dividido em cinco seções. Primeiro, há um texto introdutório sobre os temas abordados, seguido do Referencial Teórico apropriado à fase de pesquisa (dividido em Metodologia utilizada, padrão OBAA, padrão SCORM e Ferramentas de Autoria). Em terceiro acontece a apresentação da ferramenta proposta, com detalhes sobre seu projeto e desenvolvimento, bem como exemplos da sua condição de operação atual. Finalmente, esclarecem-se os resultados obtidos até o momento e conclui-se o artigo, aclarando como será a continuação do projeto. 


\section{Material e Métodos}

\subsection{Metodologia}

Até o presente momento, este trabalho baseou-se em pesquisa qualitativa pois fez-se uso de abordagens bibliográficas a fim de se compreender os conceitos a serem aplicados na ferramenta. Em seguida, este estudo torna-se aplicado, visto que almeja testar o sistema, envolvendo futuros designers instrucionais como sujeitos.

A pesquisa inicial destaca-se com estudos em websites bem como em artigos acadêmicos, objetivando o entendimento dos conceitos fundamentais conectados ao tema do projeto, como o estudo da linguagem PHP e das teorias e aplicações dos objetos de aprendizagem e seus metadados. Após, deu-se a leitura dos trabalhos de Wiley (2000) e Silva (2011), largamente citados em artigos de suas áreas, a fim de suplementar a noção dos conceitos iniciais. Também buscou-se conhecer a situação atual das ferramentas e tecnologias disponíveis para a criação e manutenção dos OAs.

O próximo passo da pesquisa foi estudo das linguagens e ferramentas compulsórias a criação do software (ênfase em PHP, JavaScript, CSS, HTML, Banco de Dados / MySQL), e, a partir do modelo proposto pela professora orientadora, deu-se início ao desenvolvimento. Adicionalmente, adotou-se o padrão OBAA para descrição de metadados através de arquivos XML e o padrão SCORM para empacotamento de arquivos.

As etapas seguintes almejam verificar o uso da ferramenta nos seguintes cenários: descrevendo um OA, empacotando um OA, procurando por um OA no repositório, etc. Para isso, adotar-se-á a participação não-observante durante a aplicação de roteiros, a fim de registrar a interação com a ferramenta. Além disso, é possível incluir registros (logs) que indicarão como e quando a ferramenta foi utilizada, fornecendo dados qualitativos e quantitativos.

\subsection{OBAA}

O OBAA é a proposta brasileira para a descrição técnica e educacional de objetos de aprendizagem através de metadados. O modelo, cuja sigla significa "Objetos de Aprendizagem Baseados em Agentes", foi desenvolvido com o objetivo de especificar padrões para requisitos técnicos $\mathrm{e}$ funcionais em resposta a problemas de interoperabilidade de alguns conteúdos digitais que, em suma, foram resolvidos com a sintaxe XML deste modelo (Silva, 2011).

O OBAA foi baseado no IEEE LOM, um padrão de referência internacional amplamente utilizado que permitia algumas modificações que eram consideradas necessárias para a criação do OBAA. Consequentemente, o OBAA agregou as categorias do LOM, aplicando modificações e adicionando novos itens (Vicari et al., 2010). Cita-se aqui: a categoria Technical do LOM foi estendida para interoperabilidade de plataformas, Accessibility foi adicionada como categoria responsável por descrever o que é necessário para utilizar o objeto e a categoria Educational do LOM recebeu novos itens como o LearningContentType, que deve especificar se o conteúdo é baseado em fatos, conceitos, experimentos ou atitudes (Silva, 2011). 


\subsection{SCORM}

O SCORM (sigla inglesa para "Modelo de Referência de Objeto de Conteúdo Compartilhável") é uma coleção de especificações e padrões que definem a interrelação de objetos de conteúdo e modelos de dados de modo que objetos são compartilháveis em sistemas que seguem este modelo (ADL, 2012). Esta especificação promove a reusabilidade, a acessibilidade, a durabilidade e a interoperabilidade de conteúdo de aprendizagem, e facilita a migração entre diferentes sistemas de gerenciamento de aprendizagem. O SCORM é responsabilidade da Advanced Distributed Learning e foi originalmente lançado em 2000, tendo como sua versão atual o SCORM 4th Edition, de 2009.

Tarouco e Dutra (2007) explicam que os recursos (também chamados mídia educacional) são a menor unidade física dentro do material SCORM. Exemplos incluem páginas web, imagens, vídeos e aplicações Flash. Sua principal característica é ser reutilizável e para que isso seja possível, usam-se os metadados. SCOs (Objetos de Conteúdo Compartilhável) são um conjunto de recursos que representa a menor unidade lógica do material: podem representar uma aula, um tópico ou um módulo de um curso. SCOs não podem ter comunicação entre si pois são independentes.

Assim, a ferramenta aqui proposta deve ser capaz de empacotar um SCO, tornando-o compartilhável em sistemas de gerenciamento de aprendizagem que utilizam SCORM, como o Moodle, por exemplo.

\subsection{Ferramentas de Autoria}

O termo ferramenta de autoria se refere a um tipo de software que objetiva a geração de objetos de aprendizagem como textos, imagens, vídeos, áudios, entre outros (Leffa, 2006). Estas ferramentas são online ou offline e têm como público-alvo estudantes e professores. O autor menciona que a preferência por estes sistemas é devido sua habilidade de oferecer um modo fácil e rápido de criar conteúdo educacional de qualidade, requerendo apenas a entrada de conhecimento e criatividade.

Dentre as vantagens das ferramentas de autoria, deve-se citar o alto nível de interação que o usuário poderá vir a ter com o objeto (tornando o aprendizado mais fácil), e o baixo custo de geração de material (Leffa, 2006; Harris, 2002). Entretanto, é importante notar que os objetos produzidos não devem substituir o professor, mas complementar o trabalho dele. HotPotatoes, Quandary, Ardora, CARLOS, CourseLab, PALOMA e eXe-Learning, são alguns exemplos.

Vale a pena destacar que a maioria destas ferramentas têm problemas de compatibilidade de hardware ou software, necessitam instalação de aplicações adicionais, não usam o modelo OBAA (ou SCORM) ou não são gratuitas.

\section{Ferramenta Proposta}

Este documento propõe uma ferramenta de autoria para objetos de aprendizagem capaz de gerar o conteúdo educacional e seus metadados no formato OBAA e realizar o empacotamento com o padrão SCORM, bem como salvar o objeto criado no repositório da própria ferramenta, conforme desejo do usuário. Sua principal característica é diferenciar-se de outras aplicações por não necessitar de nenhum tipo de instalação e ser 
5

grátis, intuitiva e simples de usar. Para isso, o sistema utiliza plataforma Web, sendo pré-requisito para sua utilização apenas um navegador com acesso à Internet.

\subsection{Projeto}

A ferramenta deve ser uma mescla de repositório com ferramenta de autoria, simples, fácil de usar e intuitiva. Para tanto, buscou-se aproveitar de uma interface limpa e amigável, bem como apresentar a possibilidade de mudar o idioma e usar uma função que automaticamente ajusta o layout de acordo com a resolução do dispositivo utilizado. Consequentemente, o usuário não deve gastar muito tempo procurando onde clicar ou o que deve ser feito. Além disso, ao mudar o layout, funções são liberadas ou escondidas, evitando, por exemplo, que alguém usando o sistema num smartphone acesse, sem intenção, a opção de criação de conteúdo educacional, que requer entrada de texto, imagens, formatação...uma tarefa que raramente será realizada através deste tipo de dispositivo.

Das funções de repositório e ferramenta de autoria, o sistema compreende quatro componentes independentes, que podem ser usados individualmente ou em conjunto, de acordo com a vontade do usuário. Tais divisões são detalhadas abaixo.

- Criação do material educacional: a ferramenta conta com um editor capaz de realizar a criação de conteúdo baseado em texto e mídia (áudio/vídeo/imagem), para o qual a saída é a tela de preenchimento de metadados. O editor pode ser acessado através da página inicial em computadores desktop e laptops.

- Preenchimento de metadados OBAA: seguinte ao uso do editor ou diretamente através da página inicial, para computadores desktop e laptops, é apresentado o formulário de metadados de acordo com o padrão OBAA. O processamento do formulário realiza a criação dos arquivos pertinentes e o empacotamento em SCORM, e armazena o objeto no banco de dados da ferramenta. Após enviar o formulário, o usuário receberá um arquivo ZIP (o Objeto de Aprendizagem propriamente dito) via download direto e será questionado se o objeto deve ser mantido ou não no repositório.

- Empacotamento SCORM: o empacotamento SCORM acontece automaticamente após enviar o formulário de metadados ou, se o OA foi previamente gerado, pode ser acessado através da página inicial em computadores desktop, laptops ou tablets. Se o último caso, será exibida uma tela na qual o usuário envia seu OA e o sistema o converte para o formato SCORM. Em ambos os casos, o OA será guardado no repositório e o usuário será indagado se deseja mantê-lo ali ou removê-lo, além de receber o OA empacotado via download direto.

- Busca: para qualquer dispositivo utilizado, pode-se realizar uma busca no banco de dados do repositório através da tela inicial. Para smartphones só é possível executar busca textual, enquanto que para tablets, laptops e computadores desktop é possível fazer uso de um menu de imagens deslizantes, representando diferentes temas-filtro para busca. A saída é uma tela exibindo os resultados da busca, com curtos resumos dos OAs encontrados, link para os detalhes de cada OA e filtros para refinar os resultados. A página de detalhes é também regida pela resolução do dispositivo utilizado: smartphones só podem ver o OA, enquanto que para todos os 
6

outros dispositivos pode-se baixar o OA com SCORM, sem SCORM ou simplesmente visualizá-lo.

\subsection{Desenvolvimento}

O processo de desenvolvimento começou com o estudo das linguagens e tecnologias necessárias: PHP, HTML, CSS, JavaScript e Banco de Dados/MySQL; bem como Programação Orientada à Objetos, Model-View-Controller e os padrões OBAA e SCORM. Em seguida, a partir do modelo proposto pela professora orientadora, desenvolveu-se protótipos de layout para os diferentes dispositivos e versões básicas das páginas mais significantes, incluindo: o formulário para descrição de metadados, o editor para criação de conteúdo educacional e a página inicial com o menu de imagens deslizante e o campo de busca textual. Em sequência, deu-se a modelagem e geração do banco de dados, seguido pela construção das classes PHP as quais seriam responsáveis pelos objetos representando os metadados e pela função de tradução. Destaca-se aqui que todos os componentes mencionados sofreram, constantemente, alterações a fim de estarem de acordo com todas as especificações e erros inesperados.

Também se trabalhou no arquivo XML através da classe PHP DOMDocument, que permitia arquivos XML de acordo com o modelo OBAA. Utilizou-se a classe ZipArchive para comprimir o arquivo XML junto com a mídia educacional e a página HTML que referencia dita mídia.

Por fim, os componentes SCORM foram implementados através de funções JavaScript e com uso da funcionalidade $\$ \_G E T$ do PHP foi realizada a programação dos filtros nos resultados da busca.

Diversos outros detalhes menores não foram, ainda, trabalhados, visto que a ferramenta encontra-se em estágio inicial de desenvolvimento.

\subsection{A Ferramenta em Funcionamento}

$\mathrm{Na}$ versão atual da ferramenta, considerada pelos autores como estágio inicial de desenvolvimento, são apresentados, completamente operantes, as seguintes funcionalidades (já descritas nas seções anteriores): formulário de metadados com geração de arquivo XML, criação do conteúdo educacional através de um editor básico, armazenamento no banco de dados, busca de termos textuais e funções de tradução e ajuste automático de layout.

As Figuras 1, 2 e 3 ilustram o funcionamento do sistema. A Figura 1 mostra a tela inicial com o sistema de busca e três botões: um para cada função da ferramenta. 
Figura 1 - Tela inicial do sistema, com as quatro funcionalidades principais do sistema.

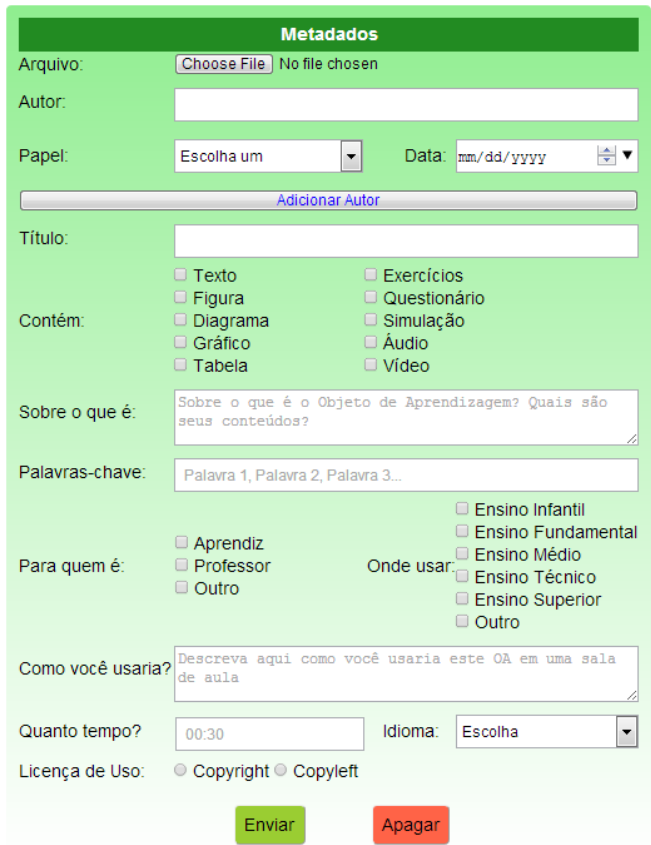

Na Figura 2 pode ser visto o formulário de metadados de acordo com o padrão

\section{FERRAMENTA de Autoria}

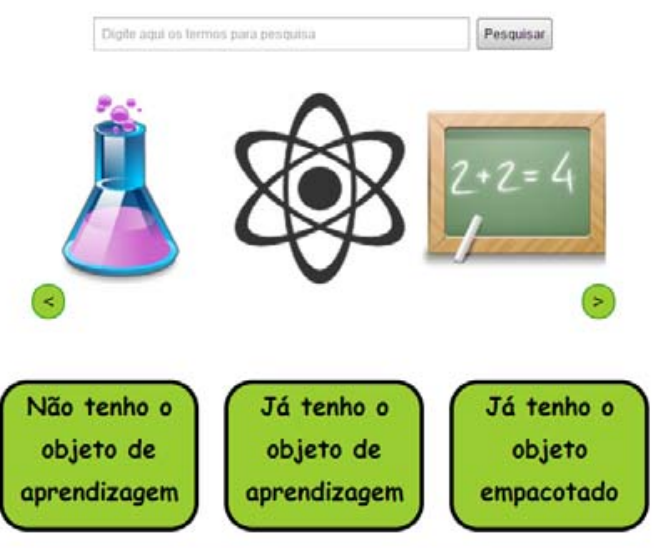

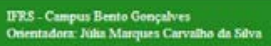

OBAA e a Figura 3 exemplifica uma busca no banco de dados, exibindo alguns detalhes dos resultados encontrados.

Figura 2 - Formulário para preenchimento dos metadados OBAA. 
Figura 3 - Tela com os resultados da busca e filtros para refinar os resultados.

\section{Resultados Parciais}

A ferramenta apresentada encontra-se em fase de desenvolvimento inicial, e, consequentemente, não foi possível valida-la completamente com casos de uso. Entretanto, durante seu desenvolvimento, foram criados alguns objetos teste, com o intuito de descobrir erros e falhas, bem como avaliar se o desempenho estava satisfatório com o projeto. A partir disso é possível destacar que, até então, a ferramenta apresenta-se fiel aos objetivos iniciais, funcionando conforme planejado.

É possível gerar mídias educacionais, assim como descrever os metadados e salvar e resgatar os objetos da base de dados. Entretanto, todas estas funções apresentam-se de forma básica, desenvolvidas, até então, apenas o suficiente para garantir seu funcionamento. O mesmo ocorre com o layout do sistema.

\section{Conclusão e Trabalhos Futuros}

As tecnologias de aprendizagem (OAs inclusos) têm se tornando um processo educacional dominante (Perera et al., 2011), e seus padrões estão, gradualmente, ganhando importância (Bohl et al., 2002), tornando imperativa a existência de ferramentas para auxiliar em seu desenvolvimento, como criação, manutenção e disseminação de OAs. Ainda mais importante é a presença de aplicações capazes de

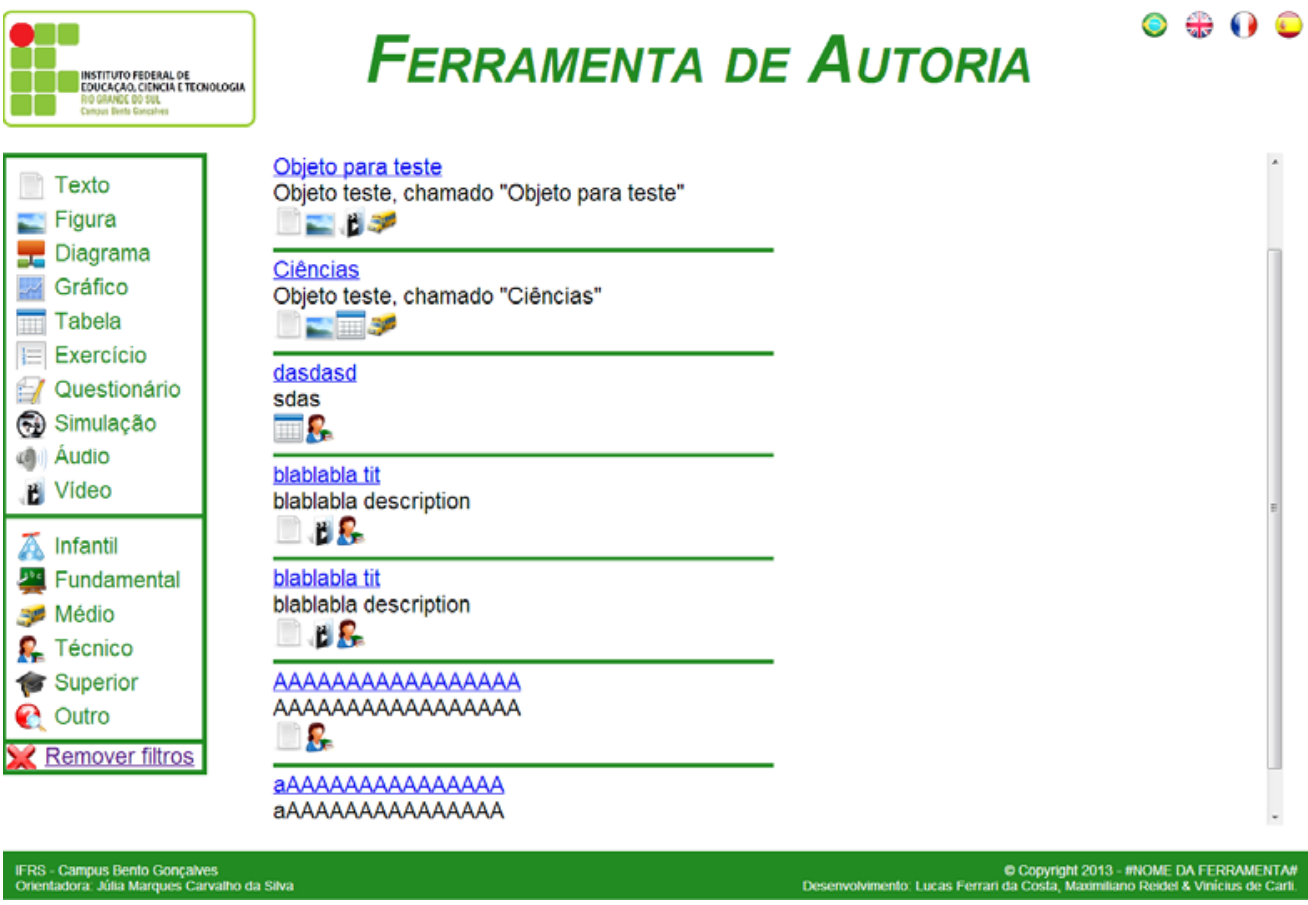

gerar objetos conforme modelos brasileiros, a fim de encorajar e difundir sua cultura. 
9

Com as características principais - busca e criação de OAs e descrição de seus metadados - já operantes, é tangível que o sistema proposto, mesmo em fase inicial de desenvolvimento, atende o perfil destacado.

Em seguida, espera-se melhorar o layout do sistema e criar a função de transformação de um OA já existente para empacotamento SCORM. Planeja-se, no futuro, aprimorar o editor e realizar testes do sistema com desingers instrucionais.

\section{Referências Bibliográficas}

ADL - Advanced Distributed Learning. SCORM. 2012. Disponível em: $<$ http://adlnet.org/scorm>. Acesso em: 20 out. 2013.

BOHL, O.; SCHEUHASE, J.; SENGLER, R.; WINAND, U. The sharable content object reference model (SCORM) - a critical review. In: ICCE 2002 Proceedings, 2002. Anais. Disponível em: <http://computer.org/csdl/proceedings/icce/2002/1509/ 00/15090950-abs.html>. Acesso em: 30 out. 2013.

HARRIS, J. An Introduction to Authoring Tools. In: ASTD's Learning Circuits, 2002. Anais. Disponível em: <http://lectora.com/downloads/AnIntroductiontoAuthoring ToolsASTD.pdf $>$. Acesso em 30 out. 2013.

LEFFA, V. J. Uma Ferramenta de autoria para o professor: o que é e o que faz. In: Letras de Hoje. Porto Alegre: PUCRS, 2006, p. 189-214.

NIENOW, A. L.; BEZ, M. R. Ferramenta de autoria para construção de objetos de aprendizagem para a área da saúde. In: Anais do VIII Seminário de Informática RS (Seminfo 2009). Anais, Ulbra.

PERERA, I.; ALLISON C.; MCCAFFERY J.; MILLER, A. Towards Effective Blended Learning with 3D MUVE - An Analysis of use case Implementations for 3D MUVE Learning. In: 3th International Conference on Computer Supported Education (CSEDU 2011). Anais. SCITEPRESS.

SILVA, J. M. C. Análise Técnica e Pedagógica de Metadados para Objetos de Aprendizagem. Porto Alegre: UFRGS, 2011. Tese de Doutorado.

TAROUCO L. M. R.; R. Dutra. Padrões e Interoperabilidade. In: PRATA, C. L.; NASCIMENTO A. C. A. A. (Ed). Objetos de Aprendizagem: Uma Proposta de Recurso Pedagógico. Brasília: MEC/SEED, 2007, p. 81-92.

WILEY, D. A. Connecting learning objects to instructional design theory: A definition, a metaphor, and a taxonomy. Livro digital, 2000. Disponível em: $<$ http://reusability.org/read/\#1>. Acesso em: 20 out. 2013.

VICARI, R. M. et al. Proposta Brasileira de Metadados para Objetos de Aprendizagem Baseados em Agentes (OBAA). Porto Alegre: UFRGS, 2010. 